\title{
Mineralocorticoid receptor is involved in rat and human ocular chorioretinopathy
}

\author{
Min Zhao,, Isabelle Célérier, ${ }^{3,4}$ Elodie Bousquet, ${ }^{1,2}$ Jean-Claude Jeanny,,2 \\ Laurent Jonet, ${ }^{1,2}$ Michèle Savoldelli,5 Olivier Offret,,2,5 Antoine Curan,,2,5 \\ Nicolette Farman, ${ }^{3,4}$ Frédéric Jaisser, ${ }^{3,4}$ and Francine Behar-Cohen 1,2,5
}

\author{
${ }^{1}$ INSERM, U872, Team 17, Centre de Recherche des Cordeliers, Paris, France. 2Université René Descartes Sorbonne Paris Cité, Paris, France. \\ 3INSERM, U872, Team 1, Centre de Recherche des Cordeliers, Paris, France. ${ }^{4}$ Université Pierre et Marie Curie Paris, Paris, France. \\ ${ }^{5}$ Assistance Publique Hôpitaux de Paris, Hôtel-Dieu de Paris, Paris, France.
}

\begin{abstract}
Central serous chorioretinopathy (CSCR) is a vision-threatening eye disease with no validated treatment and unknown pathogeny. In CSCR, dilation and leakage of choroid vessels underneath the retina cause subretinal fluid accumulation and retinal detachment. Because glucocorticoids induce and aggravate CSCR and are known to bind to the mineralocorticoid receptor (MR), CSCR may be related to inappropriate MR activation. Our aim was to assess the effect of $M R$ activation on rat choroidal vasculature and translate the results to CSCR patients. Intravitreous injection of the glucocorticoid corticosterone in rat eyes induced choroidal enlargement. Aldosterone, a specific MR activator, elicited the same effect, producing choroid vessel dilation -and leakage. We identified an underlying mechanism of this effect: aldosterone upregulated the endothelial vasodilatory $\mathrm{K}$ channel $\mathrm{KCa}$.3. Its blockade prevented aldosterone-induced thickening. To translate these findings, we treated 2 patients with chronic nonresolved CSCR with oral eplerenone, a specific MR antagonist, for 5 weeks, and observed impressive and rapid resolution of retinal detachment and choroidal vasodilation as well as improved visual acuity. The benefit was maintained 5 months after eplerenone withdrawal. Our results identify MR signaling as a pathway controlling choroidal vascular bed relaxation and provide a pathogenic link with human CSCR, which suggests that blockade of MR could be used therapeutically to reverse choroid vasculopathy.
\end{abstract}

\section{Introduction}

Glucocorticoid hormones act by binding to the glucocorticoid receptor (GR) and also to the mineralocorticoid receptor (MR), both of which belong to the nuclear receptor superfamily. Noticeably, MR has a similar high affinity for aldosterone and for the glucocorticoid hormones that largely prevail in plasma $(1,2)$. MR is typically expressed in kidney cells, in which aldosterone/MR signaling regulates sodium reabsorption $(1,2)$. MR is also present in the vasculature (endothelial and smooth muscle cells), where it contributes to the control of vascular tone $(3,4)$. The retina also has mineralocorticoid target cells. The retina is composed of the neuroretina laying on the retinal pigment epithelium (RPE), which constitutes the outer retinal barrier, controlling exchanges with the underlying choroid vessels (Supplemental Figure 1A; supplemental material available online with this article; doi:10.1172/ JCI61427DS1). We have recently shown that MR is expressed in several cell types of the neuroretina, including ganglion cells and cells of the inner nuclear layer, namely bipolar, amacrin or horizontal cells, and Muller glial cells (5); moreover, in Muller glial cells, MR controls potassium and water homeostasis through regulation of ion and water channels.

Central serous chorioretinopathy (CSCR) is characterized by focal or diffuse disruption of the outer retinal barrier, leading to subretinal serous fluid accumulation (6). CSCR affects 1 in 10,000 individuals. Recurrences and/or chronic evolution (>3

Authorship note: Min Zhao and Isabelle Célérier are co-first authors. Nicolette Farman, Frédéric Jaisser, and Francine Behar-Cohen are co-senior authors. Conflict of interest: The authors have declared that no conflict of interest exists. Citation for this article: J Clin Invest. 2012;122(7):2672-2679. doi:10.1172/JCI61427. months) occur in $30 \%-50 \%$ of cases, leading to $5 \%-10 \%$ vision loss caused by foveal thinning and intraretinal cysts, with no approved treatment currently available (6). Anti-VEGF and photodynamic therapy to target choroidal vessels are currently under evaluation for CSCR treatment, but their efficacy remains unclear (7). The mechanisms underlying CSCR are not fully understood; angiographic findings indicate that the disease may start in the choroidal vessels $(8,9)$. Optical coherence tomography (OCT), a noninvasive method of in vivo retinal imaging, has shown substantial thickening of the choroid in eyes affected by CSCR (10) and in the unaffected contralateral eye (11), which suggests that patients present a diffuse choroidal vasculopathy. Vasodilation and vascular leakage may impair hydrostatic gradients, precluding proper function of the retina and leading to retinal serous detachment. In addition, in the RPE, the transporters, pumps, ion channels, and aquaporins - or their regulatory proteins - may be functionally disturbed. Paracellular permeability through tight junctions may also be altered. However, the exact sequence of events occurring in CSCR has not yet been elucidated.

Notably, CSCR is the only choroiditis that is not only not improved, but actually worsened, by glucocorticoids $(8,12)$. Systemic and/or locoregional glucocorticoids are known risk factors for CSCR, as are endogenous cortisol levels (8). CSCR may follow psychological stress (13) and may be the presenting symptom of Cushing disease (8). However, the role of glucocorticoids in the pathogenesis of CSCR remains unknown. Based on aggravation of CSCR by glucocorticoids and the recognized MR affinity for glucocorticoids, we hypothesized that inappropriate/excessive occupancy of MR by glucocorticoids may occur in the choroid of CSCR patients. In the absence of an animal model of CSCR, we used intra- 
A

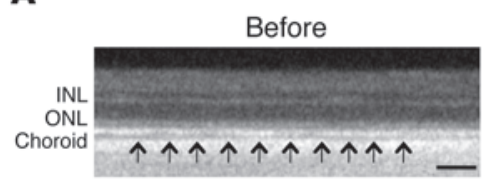

Before

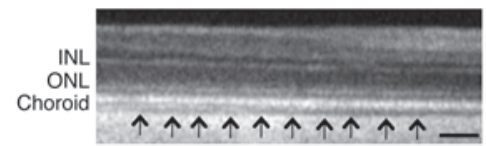

$24 \mathrm{~h}$ after corticosterone

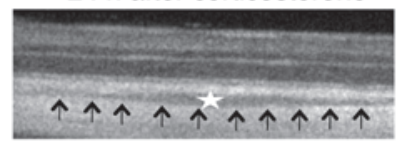

$24 \mathrm{~h}$ after aldosterone

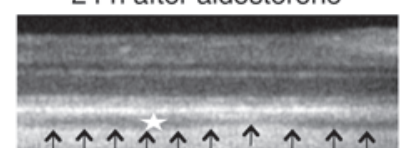

B

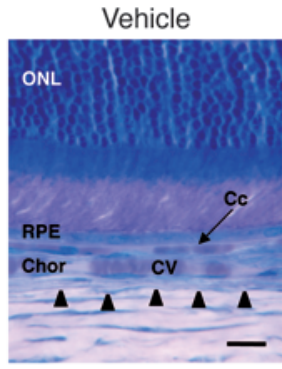

Corticosterone

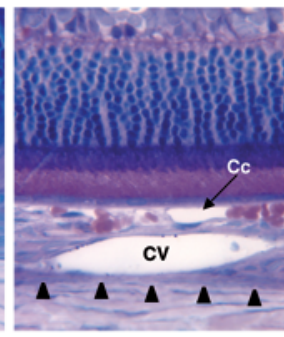

Aldosterone

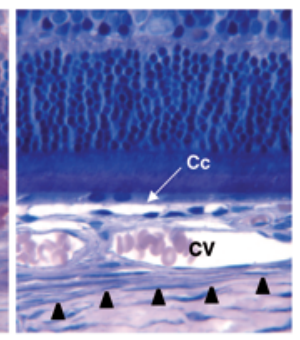

C
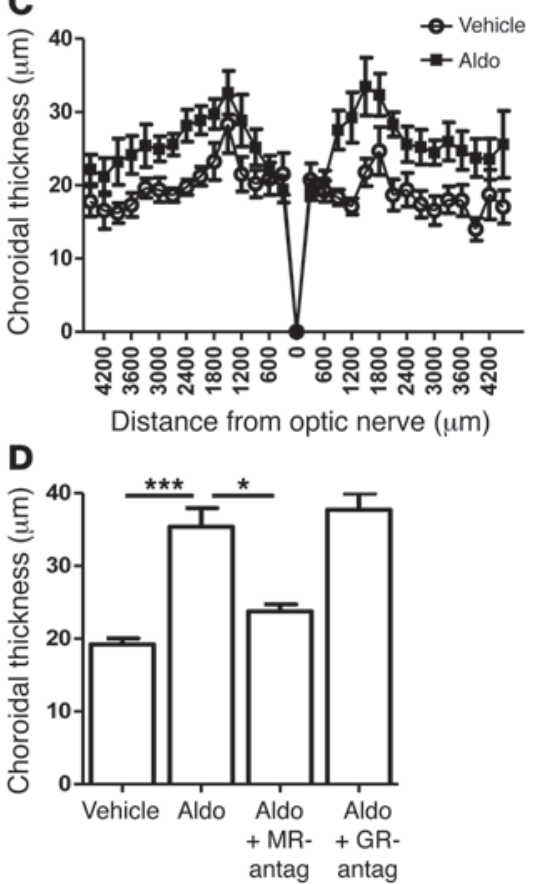

\section{Figure 1}

Induction of choroidal thickening by MR activation in rat eyes. (A) In vivo OCT scans taken in the same region of the retina before and 24 hours after IVT of high-dose corticosterone $(10 \mu \mathrm{M})$. Thickness of the choroid (arrows) was increased by corticosterone and associated with choroid vessel dilation (stars). Aldosterone $(20 \mathrm{nM})$ also enhanced choroidal thickness and vasodilation. Scale bars: $200 \mu \mathrm{m}$. (B) Historesine sections illustrating increased choroidal thickness 24 hours after $10 \mu \mathrm{M}$ corticosterone or $20 \mathrm{nM}$ aldosterone compared with vehicle-injected eyes (NaCl; $0.9 \%$ added with $0.01 \%$ ethanol). Choriocapillaries (Cc) and choroid veins (CV) were dilated in corticosterone- and aldosterone-injected eyes. Arrowheads delineate the limit of the choroid and the sclera. ONL, outer nuclear layer; Chor, choroid. Scale bar: $25 \mu \mathrm{m}$. (C) Aldosterone-induced choroidal thickening occurred all along the retina (measured on serial photographs), from the optic nerve to the periphery. Values from aldosterone-treated (Aldo) eyes were higher than those of vehicle-injected ( $\mathrm{NaCl})$ eyes $(n=12-14$ per condition). $P<0.001$, vehicle vs. aldosterone, 2-way ANOVA. (D) Quantification of choroidal thickness measured on historesine sections. Aldosterone (20 nM) induced a significant increase in choroidal thickness compared with control vehicle-injected eyes; this was significantly inhibited by coinjection of aldosterone with 500 -fold excess of a specific MR antagonist, but not a specific GR antagonist $\left(n=4-5\right.$ per condition). ${ }^{*} P<0.05,{ }^{* *} P<0.001$.

vitreous injection (IVT) of corticosteroids to characterize the shortterm (24 hours) effects of MR activation on choroidal vasculature in rat eyes. We also observed a rapid clinical benefit of treatment by an MR antagonist in patients with non-spontaneously resolved CSCR.

\section{Results}

In rat eyes, in vivo OCT of the same retinal regions before and after IVT showed that a high dose of the glucocorticoid corticosterone $(10 \mu \mathrm{M})$ induced thickening of the choroid within 24 hours (Figure 1A). This effect of corticosterone may result from its binding to GR and/or MR. The key elements supporting functional mineralocorticoid effects - the 2 steroid receptors and the glucocorticoidinactivating enzyme $11 \beta$-hydroxysteroid dehydrogenase type II (HSD2) - were detected by immunohistochemistry in choroid vessels, although HSD2 signal was low in choroid vessels (Supplemental Figure 1). MR and GR were functional, as their ligands differentially regulated gene expression (Supplemental Figure 2 and Supplemental Methods). In vivo OCT demonstrated that enhanced choroid thickness also occurred 24 hours after IVT administration of $20 \mathrm{nM}$ aldosterone, a classical MR ligand (Figure 1A). This phenotype was confirmed on histological historesine sections of the retina: thicker choroid and vasodilation of blood vessels were visible after IVT of $10 \mu \mathrm{M}$ (high-dose) corticosterone or
$20 \mathrm{nM}$ aldosterone compared with $\mathrm{NaCl}$ injection (Figure 1B). We subsequently used aldosterone as a tool for characterizing MR specificity of choroidal thickening. Choriocapillaries and choroidal vessels were dilated 24 hours after IVT aldosterone (Figure 1B), leading to choroidal thickening, whereas retinal vessels (in the inner retina) were not dilated, as visualized by lectin-stained flat-mounted retina and angiography (Supplemental Figure 3 and see below). Thus, aldosterone specifically affects the choroidal, not the retinal, vascular bed. IVT aldosterone enhanced choroidal thickness all along the retina, close to the optic nerve, as well as in the peripheral retina (Figure 1C). Aldosterone-induced choroidal thickening was blunted in the presence of the MR antagonist canrenoate, whereas the GR antagonist RU38486 was ineffective (Figure 1D), providing support for the hypothesis of an MR-mediated event. Consistent with this view, the use of low doses (100 nM, leading to limited occupancy of MR) of the glucocorticoids dexamethasone or triamcinolone acetonide - currently used for IVT in ophthalmological practice - did not induce choroidal vasodilation (data not shown). Our data therefore suggest specific regulation of the vasculature of the posterior pole of the eye by $\mathrm{MR}$, induced by the bona fide MR ligand aldosterone or by high doses of corticosterone.

Many mechanisms control vascular contractility/dilation and cross-talk between endothelial and underlying smooth muscle cells. 
A
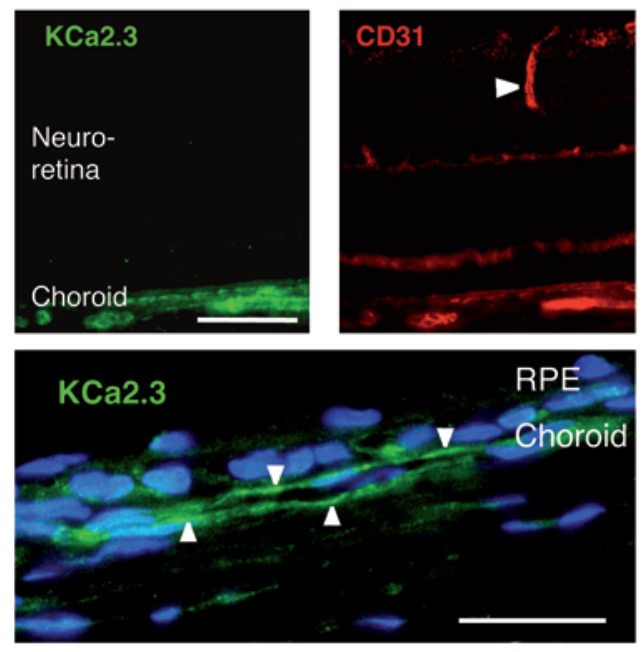

D

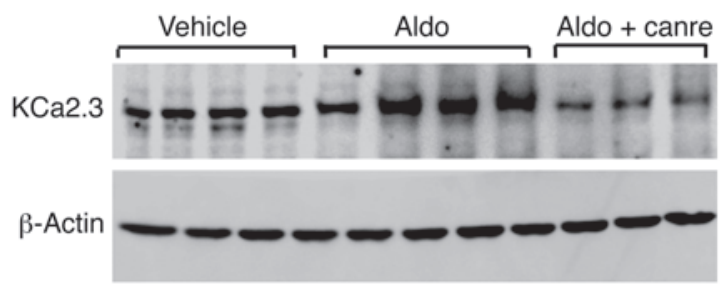

B
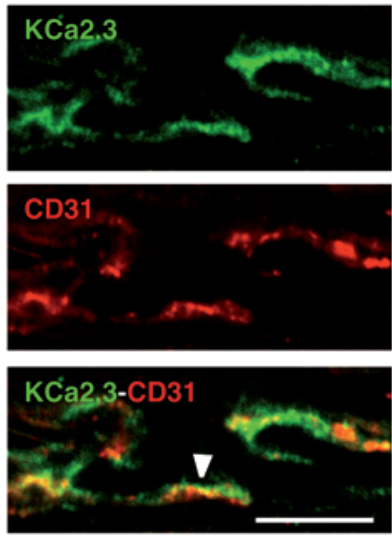

C

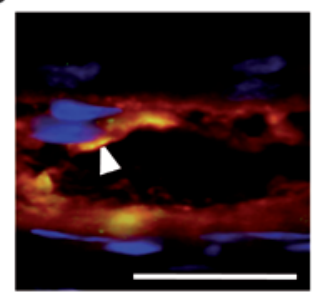

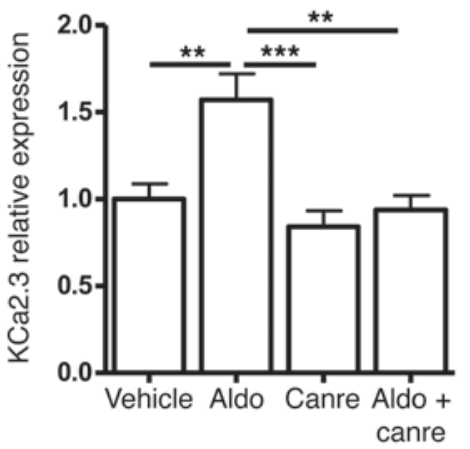

Figure 2

Expression and aldosterone regulation of $\mathrm{KCa} 2.3$ in rat eyes. (A) Immunofluorescence localization of $\mathrm{KCa} 2.3$ and $\mathrm{CD} 31$ in the retina. $\mathrm{KCa} 2.3$ labeling was restricted to the choroid vessels, CD31 labeled both choroid and retinal vessels (arrowhead). Higher magnification of KCa2.3 labeling of choroid vessels (arrowheads) and nuclei (blue) is shown below. Scale bars: $100 \mu \mathrm{m}$ (top); $25 \mu \mathrm{m}$ (bottom). (B) KCa2.3 and CD31 were colocalized (arrowhead) in choroid vessel endothelial cells. Scale bar: $25 \mu \mathrm{m}$. (C) Colocalization of KCa2.3 and CD31 (arrowhead) in a choroid vessel. Scale bar: $25 \mu \mathrm{m}$. See Supplemental Figure 4 for lower magnification and separate signals of individual markers. (D) KCa2.3 expression was increased in aldosterone-injected eyes compared with vehicle injection, an effect prevented by coinjection with the MR antagonist canreonate (Canre) in 500-fold excess. Shown are Western blot on choroid samples and quantification of KCa2.3/ $\beta$-actin, relative to vehicle-injected eyes ( $n=8$ per condition). ${ }^{* *} P<0.01,{ }^{* * *} P<0.001$.

For example, the calcium-activated $\mathrm{K}$ channel $\mathrm{KCa} 2.3$ has previously been implicated in vasodilation $(14,15)$. Since aldosterone/MR activation is known to modulate ion channel expression in other tissues (16-18), we reasoned that $\mathrm{KCa} 2.3$ might be involved in aldosterone/MR-induced choroidal vasodilation. Immunolocalization showed that $\mathrm{KCa} 2.3$ expression in rat retinas was restricted to the endothelial cells of choroid vessels (Figure 2, A-C, and Supplemental Figure 4). Western blotting of choroid samples showed a marked increase in $\mathrm{KCa} 2.3$ expression 24 hours after IVT administration of $20 \mathrm{nM}$ aldosterone, an effect that was inhibited by coinjection with the MR antagonist canrenoate (Figure 2D). Activation of the vasodilatory $\mathrm{KCa} 2.3$ channel by mineralocorticoid challenge was demonstrated by the efficacy with which apamin, a blocker of the small conductance channels KCa2.2 and KCa2.3 (the rat KCa2.1 is not sensitive to apamin), inhibited aldosterone-induced choroidal thickening and vasodilation (Figure 3 and refs. 14, 15, 19). Thus, overall, our results indicated that the mineralocorticoid hormone aldosterone promotes MR-induced choroidal thickening through a mechanism involving the $\mathrm{K}$ channel $\mathrm{KCa} 2.3$, because such an effect was abolished by a KCa blocker or by MR antagonism. As shown in Figure $3 \mathrm{~B}$, on retinal sections, aldosterone treatment enhanced the vascular area (indicative of vasodilation) as well as the extravascular area (suggestive of fluid leakage out of the vessels). In aldosteroneinjected eyes, indocyanine green (ICG) angiography allowed us to directly demonstrate in vivo the early dilation of choroid vessels, at 1 and 2.5 minutes after dye injection, and the vascular leaks, as shown by dye accumulation at 24 minutes (Figure 4).

In humans, CSCR has been shown to begin in the choroid, where focal vasodilation and leakage were observed compared with normal eyes (Supplemental Figure 5, A-C). Based on our experimental data, we hypothesized that choroid alterations in CSCR may be related, at least in part, to illicit MR activation by endogenous or exogenous glucocorticoids. To our knowledge, no treatment has yet been shown to be fully effective against forms of CSCR that do not resolve spontaneously. We hypothesized that treatment with the MR antagonist eplerenone could improve outcome in such cases.

Patient 1, a normotensive 27-year-old male of mixed European descent, was referred for impaired vision in the right eye for 1 month. 
A

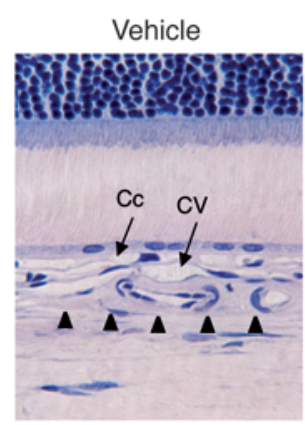

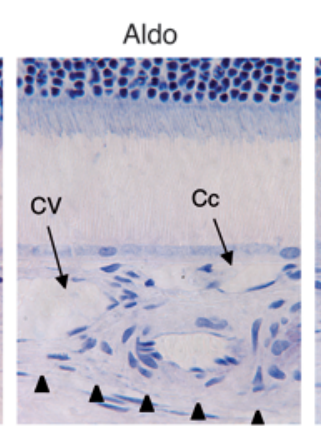

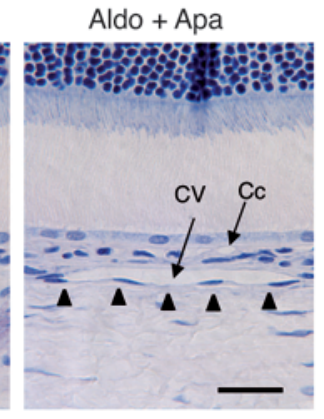

B
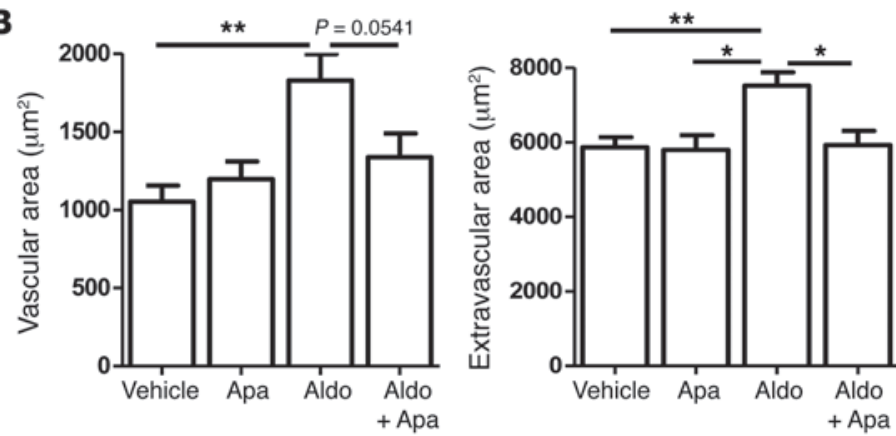
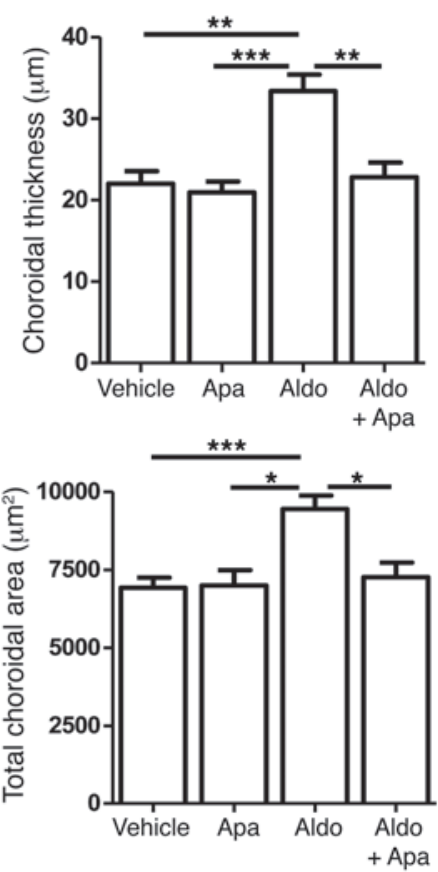

Figure 3

Aldosterone-induced choroidal thickening depends on $\mathrm{KCa} 2.3$ activity in rat eyes. (A) Historesine sections and quantification showed vasodilation of choriocapillaries and choroid veins 24 hours after aldosterone injection compared with vehicle-injected eyes ( $\mathrm{NaCl}, 0.9 \%$ added with acetic acid $0.01 \%$ ), as well as its reduction by coinjection with apamin (Apa), a specific KCa channel blocker ( $n=5-6$ per condition). Arrowheads delineate the limit of the choroid. Scale bar: $25 \mu \mathrm{m}$. (B) Quantification of vessel, extravascular, and total choroid area in tissue sections. Aldosterone injection led to a coordinate increase in area; apamin reversed this swelling, although the difference with respect to vascular area was not statistically significant $\left(n=5-11\right.$ per condition). ${ }^{\star} P<0.05,{ }^{\star \star} P<0.01,{ }^{* \star *} P<0.001$.

Visual acuity was $6 / 10$ in the right eye and 10/10 in the unaffected left eye. OCT and fluorescein angiography showed macular serous detachment caused by several disruptions of the RPE barrier and leakage points (Figure 5, A and B). No treatment was attempted. After 4 months, the macular serous detachment had worsened, and visual acuity had fallen to $5 / 10$, at which time eplerenone was prescribed. Treatment with eplerenone $(25 \mathrm{mg} / \mathrm{d}$ orally) for 1 week greatly decreased fluid accumulation beneath the retina and resulted in an increase in visual acuity to $8 / 10$. Increasing the eplerenone dose to $50 \mathrm{mg} / \mathrm{d}$ resulted in almost total resorption of subretinal fluid after
1 week, with recovery of visual acuity to $10 / 10$. The treatment was stopped after 1 month, and no recurrence had occurred 5 months later. A higher magnification of OCT oblique scans showed choroidal vessel dilation before treatment and a decrease of this dilation after 2 weeks of treatment with $50 \mathrm{mg} / \mathrm{d}$ eplerenone (Figure 5C). Blood pressure was not affected by eplerenone $(110 / 80 \mathrm{mmHg}$ before treatment; $120 / 80 \mathrm{mmHg}$ after 1 month of treatment). Patient 2 , a normotensive 56-year-old man of mixed European descent, had chronic and recurrent CSCR affecting the macula of the left eye for more than 6 years with a visual acuity of $1 / 10$. He complained that his

\section{Figure 4}

Choroid vasodilation and vascular leak after IVT aldosterone in rat eyes. After ICG injection in the tail vein, successive confocal in vivo angiography images were taken at early time points ( 1 and 2.5 minutes) and at a late phase (24 minutes) of the angiographic sequence ( $n=5$ per group). Most superficial vessels (red arrowheads) are retinal vessels; the choroid is located in the deep regions (yellow arrowheads). Aldosteroneinduced dilation of choroid, not retinal, vessels was observed at 1-2.5 minutes; at 24 minutes, the dye was washed out from vessels in the control eye, but stained focal vascular areas (arrows), attesting to the vascular leakage in aldosterone-treated eyes. Scale bar: $200 \mu \mathrm{m}$.
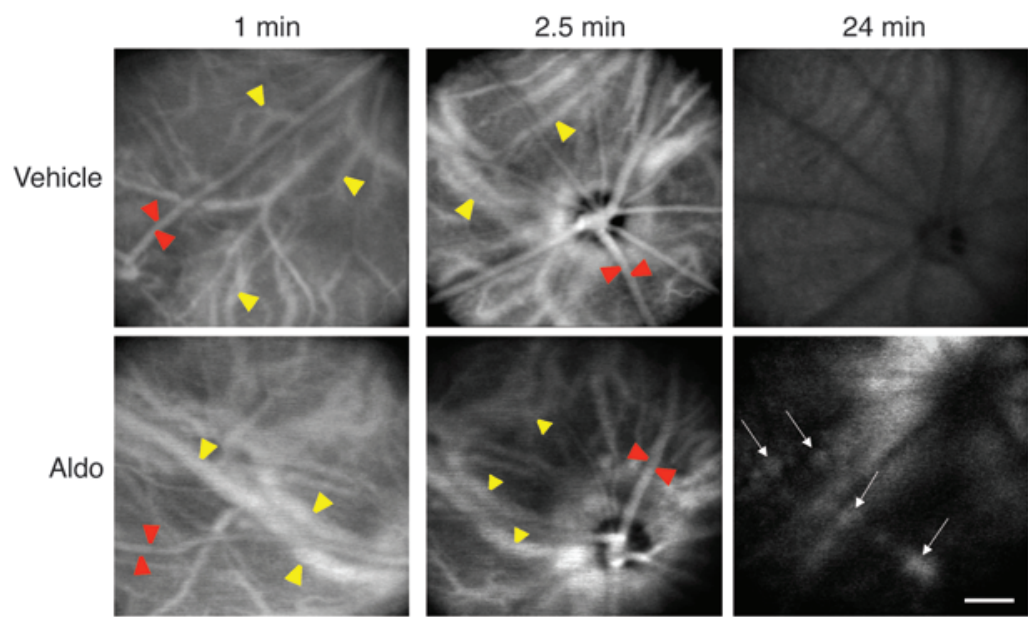
A
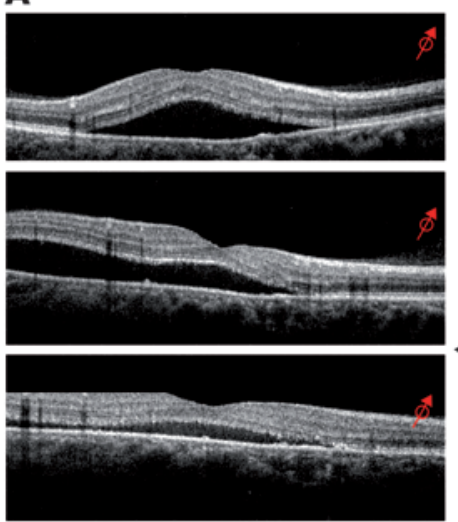

.
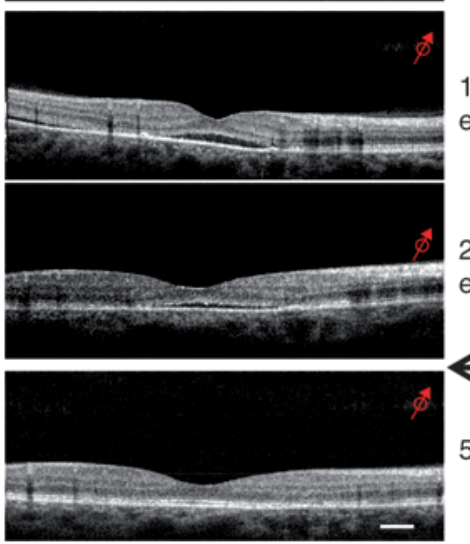

2 weeks after
1 week after

eplerenone 25 mg

4 months

before treatment

1 week

before treatment

Treatment start

1 week after

eplerenone $50 \mathrm{mg}$

eplerenone $50 \mathrm{mg}$

Treatment stopped

(after 5 weeks eplerenone)

5 months later
B
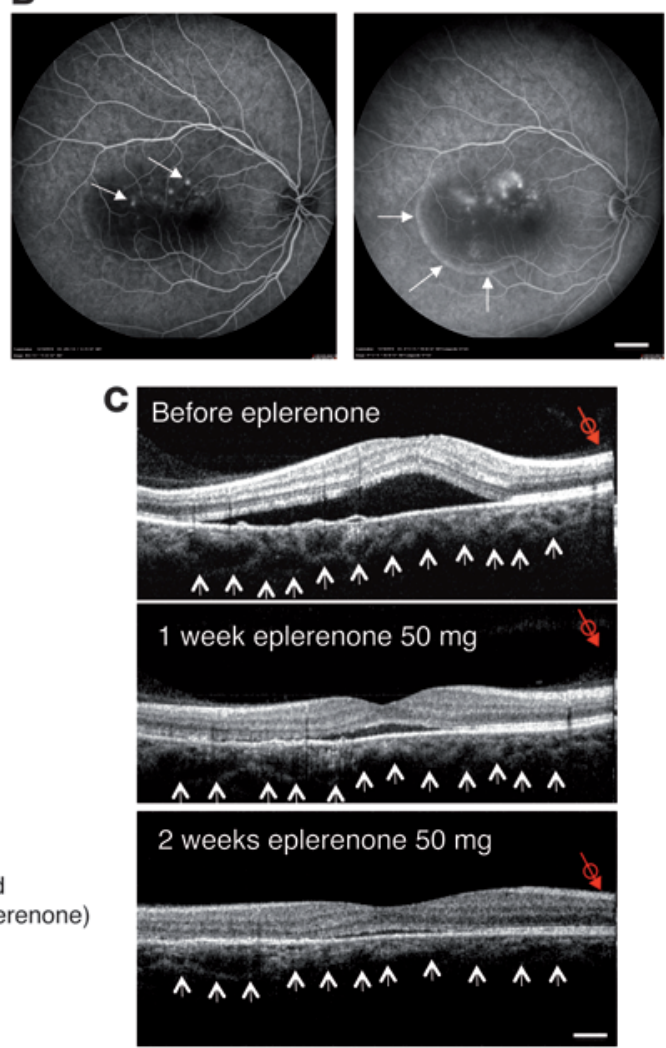

\section{Figure 5}

OCT and angiographic findings of CSCR Patient 1 before and after eplerenone treatment. (A) OCT oblique scans of the right eye. At presentation, the patient exhibited a bubble of serous retinal detachment in the macular area, whose surface increased 4 months later. Initial treatment with $25 \mathrm{mg} / \mathrm{d}$ eplerenone resulted in a rapid decrease of subretinal fluid accumulation 1 week later. Eplerenone was then increased to $50 \mathrm{mg} / \mathrm{d}$; a further reduction of the serous detachment was observed 1 week later. Near-complete resolution was observed 3 weeks after the beginning of the treatment. Eplerenone was withdrawn after 5 weeks of administration, and retinal morphology remained normal and stable 5 months after treatment interruption. Scale bar: $200 \mu \mathrm{m}$. (B) Fluorescein angiography of the right eye at presentation. The early phase of the angiogram (left) showed multiple RPE disruption white dots (arrows), and leakage at the late phase of the angiogram (right) inducing filling of the subretinal bubble with fluorescein (arrows). Scale bar: $2 \mathrm{~mm}$. (C) Choroidal thickness before and after eplerenone treatment. Other OCT oblique scans focused on deep zones of the eye (choroid vessels): at presentation, choroid vessels were dilated, which was reduced on the same oblique sections of the same zone after 1 and 2 weeks of eplerenone treatment $(50 \mathrm{mg} / \mathrm{d})$. Scale bar: $200 \mu \mathrm{m}$. Red arrows indicate the orientation and location of the captured image; small arrows delineate the lower limit of the choroid.

right eye vision dropped to $7 / 10$. OCT of the left eye showed serous fluid under the macula and intraretinal cysts (Figure 6). The right eye showed extended but discrete serous retinal detachment. The patient received $25 \mathrm{mg} / \mathrm{d}$ eplerenone for 1 week followed by $50 \mathrm{mg} / \mathrm{d}$ for $1 \mathrm{month}$. After 15 days of eplerenone at $50 \mathrm{mg} / \mathrm{d}$, both eyes showed impressive and total reduction of the subretinal fluid and disappearance of retinal cysts in the left eye. Vision returned to $10 / 10$ in the right eye and to 4/10 in the left eye, where the retina was thinned. No recurrence was observed in either eye up to 5 months after treatment arrest. Blood pressure was $115 / 85 \mathrm{mmHg}$ before treatment and remained unchanged after 1 month of eplerenone treatment. Evidence for the efficacy of eplerenone in 2 other patients with chronic CSCR is provided in Supplemental Figures 6 and 7.

\section{Discussion}

Excessive aldosterone/MR signaling may be deleterious for vessels, as impaired vascular reactivity observed in subjects with aldosterone-producing adrenal adenoma, hypertension, or heart failure can be improved by the use of MR antagonists (4, 20, 21). It has been suggested that the vascular pathological effects of aldosterone/MR result from inflammation, increased vascular reactive oxygen species, and decreased nitric oxide production $(4,22,23)$. Such mechanisms are observed in animal models when mineralocorticoid pathway activation is associated with nephrectomy and salt excess, ischemia, or pressure overload $(24,25)$. In the absence of associated pathology, enhanced mineralocorticoid activation per se may lead to distinct pathological events through other mechanisms that are not fully elucidated, such as ion channel remodeling (25). In blood vessels, both endothelial and smooth muscle MR influences the contractility properties of resistance arteries, determining arterial tone and blood pressure as well as the balance between vasoconstriction and vasodilatation $(4,26)$. MR signaling in smaller vessels, including arterioles (27) and veins (28), also contributes to vascular function regulation.

The retina is vascularized by 2 independent vascular beds, in the inner retina and in the outer retina (choroid), associated with 
Right eye
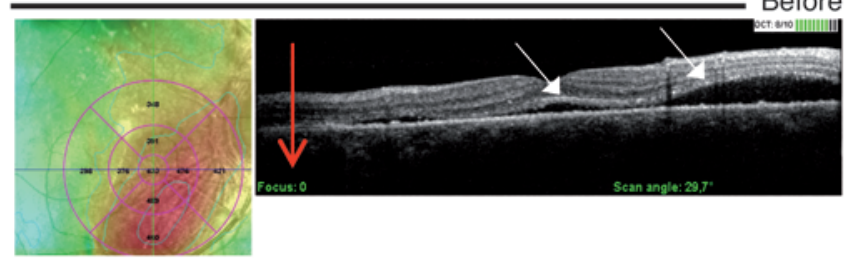

15 days eplerenone treatment
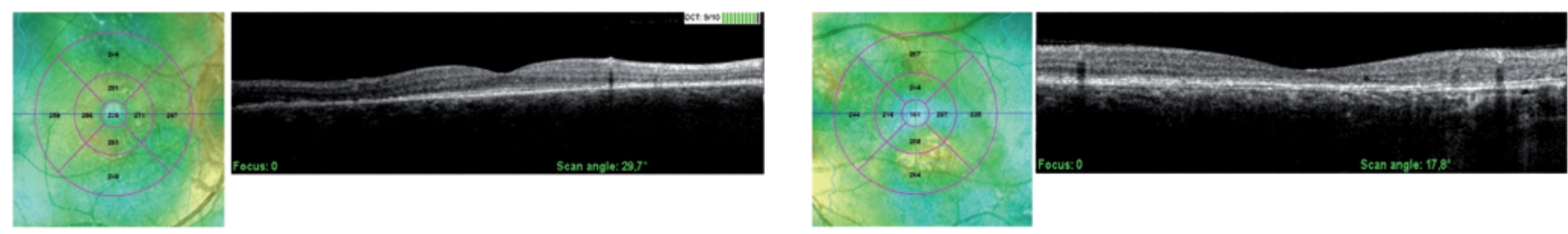

3 weeks after end of treatment
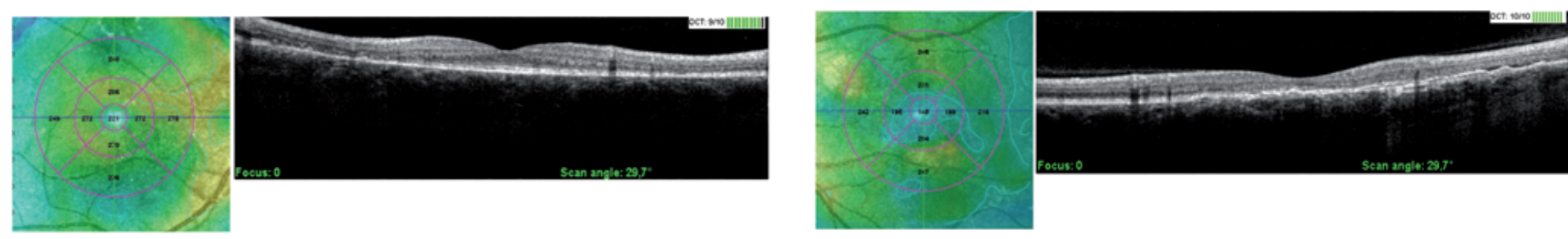

5 months after end of treatment
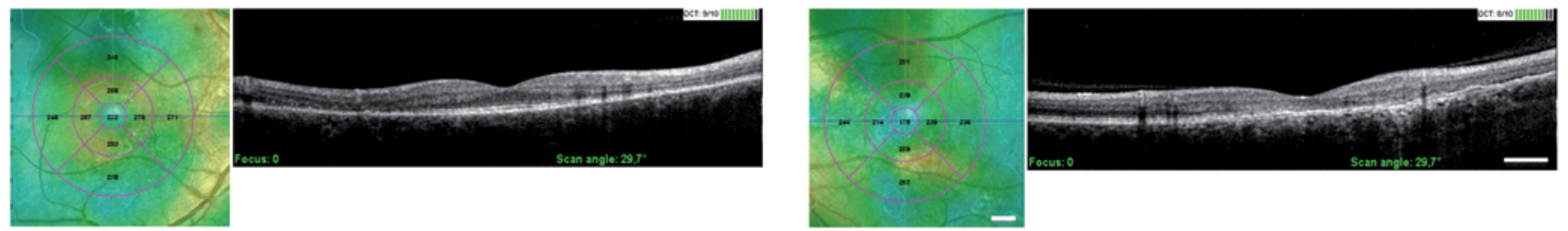

\section{Figure 6}

Kinetics of OCT vertical scans (red arrows) of CSCR Patient 2 before and after eplerenone treatment. In the right eye, OCT at presentation showed a bubble of serous retinal detachment (white arrows) close to the macula. Color mapping representation of the retinal surface showed red areas illustrating the extent of retinal detachment. Improvement was observed 15 days after beginning eplerenone treatment that remained stable at 3 weeks and 5 months after treatment was discontinued. In the left eye, OCT before treatment showed submacular fluid accumulation and intraretinal cysts (white arrows). Color mapping representation of the retinal surface showed red elevation at the center of the macula resulting from the fluid accumulation below and inside the retina. The retina recovered its normal morphology and structure with total disappearance of fluid 15 days after eplerenone treatment and 3 weeks and 5 months after eplerenone withdrawal. Scale bars: 2 mm (color mapping); $400 \mu \mathrm{m}$ (OCT).

distinct vision-threatening eye diseases. Wilkinson-Berka and colleagues first highlighted the deleterious contribution of MR overactivation in retinal vessels in an oxygen-induced retinopathy model; of note, the authors mentioned that aldosterone treatment alone did not modify retinal vessel growth (29). Our present results identified MR signaling as a pathway specifically controlling the choroidal bed, a finding we believe to be novel. In rat eyes, mineralocorticoid challenge induced choroidal vessel and choriocapillary vasodilation and focal leakage, both of which enhanced choroidal thickness.

The increased activity of the Ca-activated K channel KCa2.3 may be one mechanism involved in aldosterone-induced vasodilation in rats. The observed variation in $\mathrm{KCa} 2.3$ was relatively small at the protein level, but several events may amplify the functional response, such as traffic of the channel to the membrane, maturation, processing, activation (phosphorylation or recruitment of regulatory or accessory proteins), or channel retrieval from the membrane modulating the number of active channels. Such amplification has been highlighted previously; for example, the epithe- lial sodium channel $\mathrm{ENaC}$ is modestly upregulated 1.5 - to 3-fold by aldosterone at the protein level, whereas its activity is enhanced 50 -fold $(30,31)$. KCa2.3 channel activity is part of the endothelium-derived hyperpolarizing factor system that functionally links endothelium to smooth muscle via membrane hyperpolarization when the KCa2.3 channel is opened (32). Here, the beneficial effect of apamin, a KCa blocker, highlighted the key role of KCa channels in the aldosterone-induced choroidal vasodilation and extravascular swelling. Interestingly, we found that $\mathrm{KCa} 2.3$ was essentially expressed in the endothelium of choroidal vessels, but not in those of the inner retina, which may explain, at least in part, why MR activation did not induce any detectable effects on the retinal vessels.

Events occurring 24 hours after a single IVT dose of aldosterone in rats cannot recapitulate all CSCR features. In particular, our rat model exhibited aldosterone-induced vasodilation and fluid leakage, but no retinal detachment. Chronic MR stimulation may be required to produce serous accumulation of fluid between the neuroretina and the RPE. We observed that 3 iterative injections of aldo- 
sterone every other day led to serous retinal detachment in rat eyes (Supplemental Figure 8). However, repeated injections damaged the eye, leading to frequent bleeding, retinal tears, and cataract. In order to produce an animal model of CSCR, we anticipate that it is necessary to establish optimal procedures for in vivo intraocular delivery, namely controlled slow release of aldosterone or glucocorticoids.

In human CSCR, the occurrence of choroidal thickening in the unaffected contralateral eye could represent a precursor to retinal detachment. We propose translation of our experimental results into some human diseases with abnormal choroidal regulation of blood flow, as MR antagonism efficiently counterbalanced choroidal vasodilatation and adjacent retinal serous detachment in subjects with nonresolved CSCR. Although choroidal vessels expressed the MR-protecting enzyme HSD2, its activity may be insufficient in face of endogenous or exogenous glucocorticoid excess, a triggering factor in CSCR. As a consequence, the choroidal MR may be inappropriately activated by glucocorticoids in this disease. Illicit occupancy of MR by glucocorticoids leading to human diseases has been previously reported; the best example is the syndrome of apparent mineralocorticoid excess caused by mutations of the MR-protecting enzyme HSD2, in which patients present with hypertension that benefits from MR antagonism (33). CSCR may be another example of glucocorticoid-dependent MR activation. Whether MR variants or single nucleotide polymorphisms modifying MR activity are present in some of these patients merits future consideration. Notably, the MR antagonist eplerenone was efficient within a few days and at relatively low doses. Most interestingly, when eplerenone treatment was discontinued, the benefit was sustained for at least 5 months.

In conclusion, the results obtained in our animal studies led us to identify MR activation as a potential pathological pathway underlying the vascular choroidopathy observed in CSCR. We validated this hypothesis in humans by demonstrating that shortterm MR antagonism was an effective treatment for unresolved CSCR. Controlled clinical trials are now required to assess this therapeutic approach.

\section{Methods}

Animals. Female Lewis rats (8-12 weeks old; Janvier) were kept in pathogenfree conditions with food and water ad libitum and housed in a 12-hour light/12-hour dark cycle. For each experimental series, the number of animals is indicated in the figure legends.

Intraocular injection in rat eyes and retinal morphology. IVT was performed under ketamine-chlorpromazine anesthesia as previously described (5). Due to their hydrophobicity, injected steroids diffuse from the vitreous through the neuroretina and the choroid. The amount of hormone diffusing from the vitreous may be somewhat diluted, but we have previously shown that $20 \mathrm{nM}$ aldosterone injected IVT efficiently regulates ion/water channel expression in the neuroretina (5). We injected $5 \mu \mathrm{l}$ (i.e., $10 \%$ of the vitreous volume) of corticosterone ( 1 or $10 \mu \mathrm{M}$ final concentration) or aldosterone ( $20 \mathrm{nM}$ final concentration), alone or associated with the MR antagonist potassium canrenoate (10 $\mu \mathrm{M}$ final concentration) or with the GR antagonist RU38486 (10 $\mu \mathrm{M}$ final concentration). The KCa channel blocker apamin (10 $\mu \mathrm{M}$ final concentration) was injected 30 minutes before sacrifice. Drugs were from Sigma-Aldrich. Control eyes were injected with $5 \mu$ l solvents (see figure legends). In one experimental series, one eye only was injected with aldosterone, and this did not affect the contralateral eye (where the choroid was not thickened). In most cases, both eyes were treated similarly. See Supplemental Methods for details of flat-mount retina preparation and imaging.

In vivo assessment of rat choroid was performed on anesthetized animals using spectral domain OCT (SD-OCT; Spectralis device) adapted for small animal eyes (34). Pupils were dilated with 5\% tropicamide drops (Théa). Scans were taken in the same rats before and 24 hours after IVT. The temporal, nasal, and superior quadrants of the retina were analyzed, using the optic nerve head and the retina vessels as landmarks. Each 2-dimensional B-scan recorded at $30^{\circ}$ field of view consisted of 1,536 A-scans with an optical resolution reaching $3.5 \mu \mathrm{m}$, and the enhanced depth imaging option was used to evaluate choroidal thickness (35). Comparisons of choroid thickness were performed for a single eye before and 24 hours after injection on sections from identical regions, as visualized by simultaneous infrared imaging of retinal vessels.

In other series, rats were sacrificed by carbon dioxide inhalation, and enucleated eyes were processed for morphology, immunofluorescence, or biochemical analyses. Retinal morphology was assessed as previously described (5) on historesine sections stained with toluidine blue. Retinal layers are abbreviated in figures as follows: GCL, ganglion cell layer; INL and ONL, inner and outer nuclear layers, respectively; IS and OS, inner and outer segments of photoreceptors, respectively; Chor, choriod. Choroidal thickness was measured (ImageJ) on photographs taken in the 2,000-mm zone adjacent to the optic nerve, totaling 5 measurements on each of 5 photographs ( $328 \mu \mathrm{m}$ width) taken at $100-\mu \mathrm{m}$ intervals on each side of the optic nerve. Measurements of the vascular versus extravascular areas of the choroid were performed (using Image J) in the same photographs. In some experiments, choroidal thickness was measured all along the retina; serial photographs were taken starting from the region adjacent to the optic nerve to the periphery.

Indocyanine angiography of rat eye. ICG angiography was used to obtain images of both retinal and choroidal structures. ICG (Infracyanine, $0.5 \mathrm{mg}$ in $5 \%$ glucose; Serb) was injected in the tail vein of anesthetized rats, as it binds to albumin and therefore does not leak from normal choroidal vasculature. In vivo angiography was performed with a confocal scanning laser ophthalmoscope (HRA) using an infrared laser stimulus $(795 \mathrm{~nm}$, barrier filter at $800 \mathrm{~nm}$ ). Images were collected at various time points.

Immunolocalization. The following primary antibodies were used on cryosections: rabbit anti-KCa2.3 (1:100; Alomone Labs) associated with mouse anti-SMA (1:500; Abcam) or monoclonal antibody against rat CD31 (PECAM-1, 1:50; BD Biosciences - Pharmingen) to identify endothelial cells. The secondary antibodies were FITC-coupled goat anti-mouse IgG (1:200; Abcam) and Alexa Fluor 594-coupled goat anti-rabbit IgG (1:200; Invitrogen). Negative controls were performed by omission of primary antibody. Immunohistochemistry detection of MR, GR, and HSD2 was performed as described previously (5).

Western blot. For measurements of protein levels, the RPE-choroidsclera complex (referred to herein as choroid samples) from fresh rat eyes was dissected from the neuroretina. Samples were processed using standard methods (5) for Western blotting using rabbit anti- $\alpha \mathrm{KCa} 2.3$ (1:1,000; Alomone Labs).

CSCR cases. Patient 1 (presenting with CSCR of 4 months' duration affecting the macula of the right eye) and Patient 2 (presenting with recurrent and chronic CSCR affecting both eyes) were followed at the Ophthalmology Department of Hôtel-Dieu of Paris for at least 3 months before initiation of the treatment to assess the persistent duration of the subretinal detachment. At screening, ophthalmology surveys included best-corrected snellen visual acuity, fluorescein angiography (Spectralis HRA confocal scanning laser ophthalmoscopy), and SD-OCT (OPKO/ OTI). Individual cross-section images covered a field of $29^{\circ}$ and came with a paired scanning laser ophthalmoscopy image displaying the origin of the optical section. The adequacy of the OCT method to detect choroidal vasodilation in CSCR is demonstrated in Supplemental Figure 5. Blood analyses (kaliemia and creatinine) were performed before treatment and at 1 and 5 weeks after the treatment was started. For both patients, kaliemia 
was less than $4.5 \mathrm{mmol} / \mathrm{l}$ at all time points, and plasma creatinine was normal. Blood pressure was recorded before treatment and at 1 month.

Patients 1 and 2 were treated with oral eplerenone (Inspra; Pfizer), a selective MR antagonist, at $25 \mathrm{mg} / \mathrm{d}$ for 1 week followed by $50 \mathrm{mg} / \mathrm{d}$ for 1 month, after which treatment was discontinued. Patients 3 and 4 were treated for 3 months with eplerenone $(50 \mathrm{mg} / \mathrm{d})$.

Statistics. Data are provided as means \pm SEM. Comparison between groups was performed using 1- or 2-way ANOVA followed by Bonferroni test (GraphPad Prism 5; GraphPad Software). A $P$ value less than 0.05 was considered statistically significant.

Study approval. All experiments performed in rats were done in accordance with the European Communities Council Directive 86/609/EEC and were approved by the Animal Study Board of the Centre d'Explorations Fonctionnelles des Cordeliers (Paris, France). Experiments were performed in accordance with the ARVO statement for the Use of Animals in Opthalmic and Vision Research. Patients provided informed consent before initiation of the eplerenone treatment; the off-label prescription of eplerenone were approved by the local IRB committee (Comité de Protection des Personnes Ile de France III, Paris, France).

1. Farman N, Rafestin-Oblin ME. Multiple aspects of mineralocorticoid selectivity. Am J Physiol Renal Physiol. 2001;280(2):F181-F192.

2. Viengchareun S, Le Menuet D, Martinerie L, Munier M, Pascual-Le Tallec L, Lombes M. The mineralocorticoid receptor: insights into its molecular and (patho) physiological biology. Nucl Recept Signal. 2007;5:e012.

3. Nguyen Dinh Cat A, et al. The endothelial mineralocorticoid receptor regulates vasoconstrictor tone and blood pressure. FASEBJ. 2010;24(7):2454-2463.

4. McCurley A, Jaffe IZ. Mineralocorticoid receptors in vascular function and disease. Mol Cell Endocrinol. 2012;350(2):256-265

5 . Zhao M, et al. The neuroretina is a novel mineralocorticoid target: aldosterone up-regulates ion and water channels in Muller glial cells. FASEB J. 2010; 24(9):3405-3415

6. Gemenetzi M, De Salvo G, Lotery AJ. Central serous chorioretinopathy: an update on pathogenesis and treatment. Eye (Lond). 2010;24(12):1743-1756.

7. Bae SH, et al. A randomized pilot study of lowfluence photodynamic therapy versus intravitreal ranibizumab for chronic central serous chorioretinopathy. Am J Ophthalmol. 2011;152(5):784-792.e2.

8. Bouzas EA, Karadimas P, Pournaras CJ. Central serous chorioretinopathy and glucocorticoids. Surv Ophthalmol. 2002;47(5):431-448.

9. Pryds A, Sander B, Larsen M. Characterization of subretinal fluid leakage in central serous chorioretinopathy. Invest Ophthalmol Vis Sci. 2010; 51(11):5853-5857.

10. Imamura Y, Fujiwara T, Margolis R, Spaide RF. Enhanced depth imaging optical coherence tomography of the choroid in central serous chorioretinopathy. Retina. 2009;29(10):1469-1473.

11. Maruko I, Iida T, Sugano Y, Ojima A, Sekiryu T. Subfoveal choroidal thickness in fellow eyes of patients with central serous chorioretinopathy. Retina. 2011;31(8):1603-1608.

12. Carvalho-Recchia CA, et al. Corticosteroids and central serous chorioretinopathy. Ophthalmology. 2002;109(10):1834-1837.

13. Haimovici R, Koh S, Gagnon DR, Lehrfeld T,

\section{Acknowledgments}

The authors thank INSERM, the Assistance-Publique-Hopitaux de Paris at Hotel-Dieu, the Union Nationale des Aveugles et Deficients Visuels, the European section of the Aldosterone Council, the Leducq Foundation, and the Agence Nationale de la Recherche (ANR Mirendo and Mineraloret) for financial support. SD-OCT was performed on rat eyes at the Centre d'Explorations Fonctionnelles of Centre de Recherche des Cordeliers. We thank Nicolas Marsollier for critical reading of the manuscript. I. Célérier is the recipient of a PHD grant from Ministere de la Recherche. M. Zhao is supported by INSERM.

Received for publication October 13, 2011, and accepted in revised form May 2, 2012.

Address correspondence to: Nicolette Farman, INSERM U872, equipe 1, Centre de Recherche des Cordeliers, 15 rue de l'Ecole de Médecine, 75006 Paris, France. Phone: 0033144278104; Fax: 003344276421; E-mail: nicolette.farman@crc.jussieu.fr.
Wellik S. Risk factors for central serous chorioretinopathy: a case-control study. Ophthalmology. 2004;111(2):244-249.

14. Burnham MP, et al. Characterization of an apamin-sensitive small-conductance $\mathrm{Ca}(2+)$ activated $\mathrm{K}(+)$ channel in porcine coronary artery endothelium: relevance to EDHF. Br J Pharmacol. 2002;135(5):1133-1143.

15. Brahler S, et al. Genetic deficit of SK3 and IK1 channels disrupts the endothelium-derived hyperpolarizing factor vasodilator pathway and causes hypertension. Circulation. 2009;119(17):2323-2332.

16. Escoubet B, Coureau C, Bonvalet JP, Farman $\mathrm{N}$. Noncoordinate regulation of epithelial $\mathrm{Na}$ channel and $\mathrm{Na}$ pump subunit mRNAs in kidney and colon by aldosterone. Am J Physiol. 1997; 272(5 pt 1):C1482-C1491.

17. Wald H, Garty H, Palmer LG, Popovtzer MM. Differential regulation of ROMK expression in kidney cortex and medulla by aldosterone and potassium. Am J Physiol. 1998;275(2 pt 2):F239-F245.

18. Benitah JP, Perrier E, Gomez AM, Vassort G. Effects of aldosterone on transient outward $\mathrm{K}+$ current density in rat ventricular myocytes. J Physiol. 2001; 537(pt 1):151-160.

19. Weatherall KL, Seutin V, Liegeois JF, Marrion NV. Crucial role of a shared extracellular loop in apamin sensitivity and maintenance of pore shape of small-conductance calcium-activated potassium (SK) channels. Proc Natl Acad Sci U S A. 2011; 108(45):18494-18499.

20. Struthers AD. Aldosterone-induced vasculopathy. Mol Cell Endocrinol. 2004;217(1-2):239-241.

21. Funder JW. Aldosterone and mineralocorticoid receptors in the cardiovascular system. Prog Cardiovasc Dis. 2010;52(5):393-400.

22. Brown NJ. Aldosterone and vascular inflammation. Hypertension. 2008;51(2):161-167.

23. Schiffrin EL. Effects of aldosterone on the vasculature. Hypertension. 2006;47(3):312-318

24. Griol-Charhbili V, Fassot C, Messaoudi S, Perret C, Agrapart V, Jaisser F. Epidermal growth factor receptor mediates the vascular dysfunction but not the remodeling induced by aldosterone/salt. Hypertension. 2011;57(2):238-244.

25. Messaoudi S, Azibani F, Delcayre C, Jaisser F. Aldosterone, mineralocorticoid receptor, and heart failure. Mol Cell Endocrinol. 2012;350(2):266-272.

26. Leopold JA, et al. Aldosterone impairs vascular reactivity by decreasing glucose-6-phosphate dehydrogenase activity. Nat Med. 2007;13(2):189-197.

27. Heylen E, Huang A, Sun D, Kaley G. Nitric oxidemediated dilation of arterioles to intraluminal administration of aldosterone. J Cardiovasc Pharmacol. 2009;54(6):535-542.

28. Bafford R, et al. Mineralocorticoid receptor expression in human venous smooth muscle cells: a potential role for aldosterone signaling in vein graft arterialization. Am J Physiol Heart Circ Physiol. 2011;301(1):H41-H47.

29. Wilkinson-Berka JL, Tan G, Jaworski K, Miller AG. Identification of a retinal aldosterone system and the protective effects of mineralocorticoid receptor antagonism on retinal vascular pathology. Circ Res. 2009;104(1):124-133.

30. Frindt G, Ergonul Z, Palmer LG. Surface expression of epithelial Na channel protein in rat kidney. J Gen Pbysiol. 2008;131(6):617-627.

31. Soundararajan R, Pearce D, Hughey RP, Kleyman TR. Role of epithelial sodium channels and their regulators in hypertension. J Biol Chem. 2010; 285(40):30363-30369.

32. Edwards G, Feletou M, Weston AH. Endothelium-derived hyperpolarising factors and associated pathways: a synopsis. Pflugers Arch. 2010; 459(6):863-879.

33. White PC, Mune T, Agarwal AK. 11 beta-Hydroxysteroid dehydrogenase and the syndrome of apparent mineralocorticoid excess. Endocr Rev. 1997; 18(1):135-156.

34. Fischer MD, et al. Noninvasive, in vivo assessment of mouse retinal structure using optical coherence tomography. PLoS One. 2009;4(10):e7507.

35. Spaide RF, Koizumi H, Pozzoni MC. Enhanced depth imaging spectral-domain optical coherence tomography. Am J Ophthalmol. 2008;146(4):496-500. 